\title{
Interaction potential between two spheres mediated by excluded volume polymers
}

\author{
R. Tuinier, ${ }^{1,2}$ H. N. W. Lekkerkerker, ${ }^{1, *}$ and D. G. A. L. Aarts ${ }^{1}$ \\ ${ }^{1}$ Van 't Hoff Laboratory, Debye Research Institute, University of Utrecht, Padualaan 8, NL-3584 CH Utrecht, The Netherlands \\ ${ }^{2}$ Forschungszentrum Jülich, Institut für Festkörperforschung, Weiche Materie, D-52425 Jülich, Germany
}

(Received 23 October 2001; published 20 June 2002)

\begin{abstract}
The interaction between two spheres in a solution of nonadsorbing polymers, with excluded volume interaction, is calculated from the depletion layers around the spheres using the generalized Gibbs adsorption equation. By combining the bulk correlation length with the curvature-dependent interfacial tension between a sphere and the surrounding polymer solution [Hanke, Eisenriegler, and Dietrich, Phys. Rev. E 59, 6853 (1999)], the depletion layer thickness around a sphere is obtained. The resulting contact potential agrees with a scaling prediction of de Gennes in the semidilute regime.
\end{abstract}

DOI: 10.1103/PhysRevE.65.060801

PACS number(s): 61.25.Hq, 82.70.Dd

Polymer-mediated attraction between colloidal particles was first demonstrated theoretically by Askura and Oosawa (AO) $[1,2]$ and later, independently by Vrij [3]. Their theory applies to a mixture consisting of hard spheres, with radius $R$, and ideal polymer chains, characterized by a radius of gyration $R_{g}$, in a background solvent. The so-called AO theory gives good predictions as long as the radius of gyration of the polymer is significantly smaller than the sphere radius. If the depletion-induced attraction is sufficiently strong, mixing hard colloidal spheres and nonadsorbing polymer leads to macroscopic phase instability [4-9]. This has driven the extension of the theory towards predictions of the phase behavior, first using a perturbative approach [10] and later using an osmotic equilibrium theory $[11,12]$. The theory of Lekkerkerker et al. [12], which takes into account the polymer partitioning between the phases, has been verified for small polymer-to-colloid size ratios by experiment [13] and computer simulation results $[14,15]$.

For large spheres immersed in a semidilute polymer solution, where the characteristic polymer length scale is the correlation length $\xi$, de Gennes [16] derived a scaling expression for the contact potential

$$
\beta W(0)=-\frac{R}{\xi} .
$$

where $\beta=1 / k T$.

Under conditions where $R_{g} \geqslant R$, which involves many practical systems such as protein-polysaccharide mixtures [17-20], the AO theory is known to fail. For such small spheres, the free energy of immersion of a sphere, $F$, is proportional to the polymer concentration $n_{p}$ (number density) times the sphere volume $\sim n_{p} R^{3}$ [16]. In a semidilute polymer solution $\xi \sim n_{p}^{-3 / 4}$, so the free energy of immersion turns to $F \sim(\xi / R)^{-4 / 3}$, leading to

$$
\beta W(0)=-\left(\frac{R}{\xi}\right)^{4 / 3}
$$

\footnotetext{
*Corresponding author.

Email address: h.n.w.lekkerkerker@chem.uu.nl
}

Odijk [21] rederived this equation by noting that the depletion layer around a small sphere is of the order of the sphere radius. Following de Gennes [16] and Odijk [21], the extreme case $\xi / R \rightarrow \infty$ has been studied thoroughly by various workers in the past few years [22-27]. The situation where the radius of gyration (or for the semidilute case the correlation length) is of the order of the sphere radius remains a significant problem. As the polymer length scale increases with respect to the sphere radius the depletion interaction becomes weaker and the onset of phase separation shifts to larger polymer concentrations. At sufficiently high polymer concentration the typical length scale is the correlation length rather than the polymer's radius of gyration and the depletion layer thickness becomes concentration dependent [28]. Moreover, the polymer osmotic pressure then significantly deviates from Van 't Hoff's law. Therefore for the situation of $R_{g} \geqslant R$ taking into account the excluded volume interaction between polymer segments becomes essential especially when phase transitions take place around or above the polymer overlap concentration.

Here a simple theory is presented that describes depletion interaction up to at least a size ratio of unity, and in which the excluded volume effect of the polymer segments is taken into account. For the calculation of the pair potential between two particles we use the adsorption method [29,30], which follows from the generalized Gibbs adsorption equation,

$$
-\left(\frac{\partial W(h)}{\partial \mu}\right)_{h}=\Gamma(h)-\Gamma(\infty) .
$$

Here, $\mu$ is the chemical potential of the polymers and $\Gamma(h)$ is the adsorption when the particles are a distance $h$ apart (for two spheres this is the center to center distance minus the sphere diameter). To use this method, which has proven to be very efficient in the calculation of the depletion-induced pair potential (see for instance Refs. [31-33]), we must have an expression for the chemical potential as well as for the (negative) adsorption.

The adsorbed amount depends on the polymer segment concentration profiles around the two colloidal particles. It has been shown [32-34] that the density around two particles is reproduced very well when the product of the den- 
sities around the single particles is taken. Thus within the accuracy of the product function approximation, the profile around a single particle, captured by the immersion free energy, yields the interaction potential. The depletion layer thickness around a sphere in a dilute polymer solution with excluded volume interaction follows from the interfacial tension. The flat and curvature-dependent terms of this interfacial tension were calculated by Hanke, Eisenriegler, and Dietrich [35]. Taking a step function for the polymer concentration profile around a sphere leads to the following expression for the depletion layer thickness $\Delta$ up to third order in $R_{g} / R$ :

$$
\Delta=R\left\{\left[1+3 A \frac{R_{g}}{R}+3 B\left(\frac{R_{g}}{R}\right)^{2}+3 C\left(\frac{R_{g}}{R}\right)^{3}\right]^{1 / 3}-1\right\},
$$

where $\quad A=(2 / \sqrt{\pi})\{1-[1-(3 \ln 2 / 2)-\pi / 2+\pi / \sqrt{3}] / 4\}$ $\approx 1.071, \quad B=1-5 \pi / 8+\frac{17}{36}+\pi \sqrt{3} / 4 \approx 0.8691, \quad$ and $C=$ $-\left(1673 \pi / 48-\frac{551}{15}-40 \pi / \sqrt{3}\right) / 3 \sqrt{\pi} \approx-0.03992$. In order to obtain the polymer concentration dependence of the depletion layer thickness, we make the assumption that Eq. (4) holds but with the correlation length $\xi$ replacing the polymer radius of gyration $R_{g}$. The correlation length $\xi$ is taken from renormalization group theory [[36]; Eq. (19.24)]. The adsorption in the space surrounding two colloidal hard spheres can now be directly computed from the overlap volume of the depletion zones:

$$
\Gamma\left(h, n_{p}\right)-\Gamma\left(\infty, n_{p}\right)=\frac{2}{3} \pi n_{p} \Delta^{3}\left(1-\frac{h}{2 \Delta}\right)^{2}\left(2+\frac{3 R}{\Delta}+\frac{h}{2 \Delta}\right) .
$$

for $h \leqslant 2 \Delta$ and $\Gamma(h)=\Gamma(\infty)$ for $h>2 \Delta$.

Rewriting Eq. (3) using the Gibbs-Duhem relation $n_{p}^{-1} d \Pi=d \mu$ yields

$$
W(h)=-\int_{0}^{n_{p}} d n_{p}^{\prime} \frac{1}{n_{p}^{\prime}}\left(\frac{\partial \Pi}{\partial n_{p}^{\prime}}\right)\left[\Gamma\left(h, n_{p}^{\prime}\right)-\Gamma\left(\infty, n_{p}^{\prime}\right)\right] .
$$

Using the renormalization group expression for the osmotic compressibility [[36]; Eq. (17.53)]

$$
\frac{\partial(\beta \Pi)}{\partial n_{p}}=1+2.63 \frac{n_{p}}{n_{p}^{*}}\left(\frac{1+3.25 \frac{n_{p}}{n_{p}^{*}}+4.15\left(\frac{n_{p}}{n_{p}^{*}}\right)^{2}}{1+1.48 \frac{n_{p}}{n_{p}^{*}}}\right)^{0.309}
$$

allows to calculate $W(h)$.

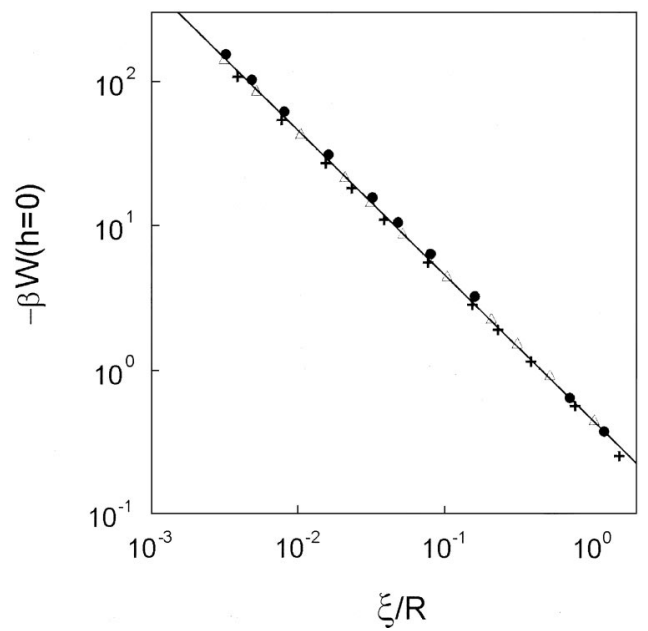

FIG. 1. Interaction potential contact value for two spheres in a semidilute polymer solution as a function of the correlation length normalized with the sphere radius. The curve corresponds to Eq. (1), a scaling result from de Gennes [16] for relatively large spheres. The symbols refer to our results for $n_{p} / n_{p}^{*}=3(\bullet), 5(\Delta)$, and $7(+)$.

We now compare our theory for the depletion potential between two spheres in a polymer solution with excluded volume interaction with de Gennes' scaling prediction Eq. (1). Results for $\beta W(0)$ obtained using Eq. (6) given above for three polymer concentrations in the semidilute regime, $n_{p} / n_{p}^{*}=3,5$, and 7 (symbols), are plotted in Fig. 1 as a function of $\xi / R$. A first observation that can be made is that the data collapse onto a single curve underlining the scaling assumption of de Gennes [16] that only the two length scales $\xi$ and $R$ are relevant and that the minimum of the potential in the semidilute regime only depends on the polymer concentration through (the concentration-dependent) $\xi$. The scaling predictions of Eq. (1) is also indicated in the plot, and a best fit gave $\beta W(0)=-0.45 R / \xi$. It is remarkable that up to $\xi$ $=R$, Eq. (1) describes the results extremely well. In order to compare with the potential between smaller spheres, the higher order terms in Eq. (4) are required. It is thus demonstrated how well our simple theory corresponds to the scaling theory of de Gennes [16].

Part of this work was supported by the Council for Chemical Sciences of the Netherlands Organization for Scientific Research and Unilever Research Vlaardingen. M. E. Cates, M. Fuchs, and E. Eisenriegler are thanked for their valuable suggestions and help. We are indebted to A. A. Louis for sending us a very useful preprint.
[1] S. Asakura and F. Oosawa, J. Chem. Phys. 22, 1255 (1954).

[2] S. Asakura and F. Oosawa, J. Polym. Sci. 33, 183 (1958).

[3] A. Vrij, Pure Appl. Chem. 48, 471 (1976).

[4] C. F. Vester, Kolloid-Z. 84, 63 (1938).

[5] R. Li-In-On, B. Vincent, F. A. Waite, ACS Symp. Ser. 9, 165 (1975).
[6] C. Cowell, R. Li-In-On, B. Vincent, and F. A. Waite, J. Chem. Soc., Faraday Trans. 74, 337 (1978).

[7] B. Vincent, P. F. Luckham, and F. A. Waite, J. Colloid Interface Sci. 73, 508 (1980).

[8] H. De Hek and A. Vrij, J. Colloid Interface Sci. 70, 592 (1979).

[9] P. R. Sperry, J. Colloid Interface Sci. 87, 375 (1982); 99, 97 
(1984); P. R. Sperry, H. B. Hopfenberg, and N. L. Thomas, ibid. 82, 62 (1981).

[10] A. P. Gast, C. K. Hall, and W. B. Russel, J. Colloid Interface Sci. 96, 251 (1983).

[11] H. N. W. Lekkerkerker, Colloids Surface 51, 419 (1990).

[12] H. N. W. Lekkerkerker, W. C. K. Poon, P. N. Pusey, A. Stroobants, and P. B. Warren, Europhys. Lett. 20, 559 (1992).

[13] S. M. Ilett, A. Orrock, W. C. K. Poon, and P. N. Pusey, Phys. Rev. E 51, 1344 (1995).

[14] E. J. Meijer and D. Frenkel, J. Chem. Phys. 100, 6873 (1994).

[15] M. Dijkstra, J. M. Brader, and R. Evans, J. Phys.: Condens. Matter 11, 10079 (1999).

[16] P. G. de Gennes, C.R. Seances Acad. Sci., Ser. B 288, 359 (1979).

[17] V. Ya. Grinberg and V. B. Tolstoguzov, Food Hydrocolloids 11, 145 (1997).

[18] A. Syrbe, W. J. Bauer, and H. Klostermeyer, Int. Dairy J. 8, 179 (1998).

[19] A. M. Kulkarni, A. P. Chatterjee, K. S. Schweizer, and C. F. Zukoski, Phys. Rev. Lett. 83, 4554 (1999).

[20] J.-L. Doublier, C. Garnier, C. Renard, and C. Sanchez, Curr. Opin. Colloid Interface Sci. 5, 184 (2000).

[21] T. Odijk, Macromolecules 29, 1842 (1996).

[22] T. Odijk, J. Chem. Phys. 106, 3402 (1997).
[23] H. M. Schaink and J. A. M. Smit, J. Chem. Phys. 107, 1004 (1997).

[24] E. Eisenriegler, A. Hanke, and S. Dietrich, Phys. Rev. E 54, 1134 (1996).

[25] E. Eisenriegler, J. Chem. Phys. 113, 5091 (2000).

[26] R. P. Sear, Phys. Rev. E 58, 724 (1998).

[27] R. P. Sear, J. Chem. Phys. 115, 575 (2001).

[28] P. G. Bolhuis, A. A. Louis, J. P. Hansen, and E. J. Meijer, J. Chem. Phys. 114, 4296 (2001).

[29] D. G. Hall, J. Chem. Soc., Faraday Trans. 68, 2169 (1972).

[30] S. G. Ash, D. H. Everett, and C. Radke, J. Chem. Soc., Faraday Trans. 69, 1256 (1973).

[31] Y. Mao, P. Bladon, H. N. W. Lekkerkerker, and M. E. Cates, Mol. Phys. 92, 151 (1997).

[32] R. Tuinier, G. A. Vliegenthart, and H. N. W. Lekkerkerker, J. Chem. Phys. 113, 10768 (2000).

[33] R. Tuinier, G. A. Vliegenthart, and H. N. W. Lekkerkerker, Macromolecules 34, 4636 (2001).

[34] M. S. Wertheim, L. Blum, and D. Bratko, Micellar Solutions and Microemulsions, edited by S. H. Chen and R. Rajagopalan (Springer, New York, 1990), pp. 99-110.

[35] A. Hanke, E. Eisenriegler, and S. Dietrich, Phys. Rev. E 59, 6853 (1999).

[36] L. Schäfer, Excluded Volume Effects in Polymer Solutions (Springer-Verlag, Berlin, 1999), Chaps. 17-19. 